\section{Co-directores}

Marc Antoni Broggi i Trias (PCBC)

Francesc Borrell (UB)

\section{Jefa de Redacción}

Núria Estrach (UAB)

\section{Consejo científico}

Juan Carlos Hernández Clemente

Juan Medrano Albéniz

Vicente Morales Hidalgo

\section{Correspondencia}

Web:

http://www.fundacionletamendi.com

Correo electrónico:

info@fundacionletamendi.com

\section{Envío de manuscritos:}

http://www.fundacionletamendi.com/revista-foliahumanistica/envio-de-manuscritos/

\begin{abstract}
Información editorial
Folia Humanística publica artículos por encargo solicitados a especialistas, así como aquellas propuestas enviadas por los autores y aceptadas tras su evaluación por pares de académicos especializados.

Los textos recibidos se publicarán en la lengua original (castellano, catalán, inglés y francés); los que se consideren de relevancia mayor serán traducidos al inglés y castellano.

Los artículos deben ser originales y acompañados del documento "derechos de autor" que encontrarán en la web, junto a las normas de presentación a seguir.

Cada artículo publicado al final tendrá especificado la referencia de citación, donde se incluirá el número DOI ${ }^{\circledR}$.

\section{Distribución \\ La Revista Folia Humanística es de libre acceso a consultar online. \\ http://www.fundacionletamendi.com/category/rev istal}

Folia Humanística es una revista internacional que tiene el doble objetivo de fomentar, por un lado, la reflexión y el debate público en el ámbito de la Salud, Ciencias Sociales y Humanidades, y por el otro, la colaboración entre distintos equipos de investigación nacionales e internacionales que dinamicen el diálogo entre la filosofía de la medicina, la salud pública y la justicia social. Dividida en "Tema del día", (artículos para el debate), "Pensamiento actual", (artículos críticos de novedades editoriales), y "Arte, Salud y Sociedad", la revista se esfuerza en fortalecer las conexiones entre la investigación académica, la práctica clínica, las experiencias de los pacientes y sus implicaciones éticas y estéticas en la sociedad. Todo ello con la intención de favorecer la reflexión entre diferentes disciplinas sobre temas de actualidad y las tendencias más novedosas en el campo de las Humanidades y la Salud.

Folia Humanística is an International Journal, born with the dual aim of fuelling the discussion and public debate on issues of health, social sciences and humanities and on the hand, of fostering cooperation between various research groups, both national and International, to spur the dialogue between philosophy and medicine, public health and social justice. The Journal is divided into three different sections: "main focus" (article for debate), "Contemporary thought" (critical reviews of new Publications) and "Arts, Health and Society" which all contribute to strengthening the links between academic research, clinical practice, the experience of patients and their ethical and esthetical implications for society. Ultimately, the intention of the Journal is to promote reflection at the crossroads of several disciplines on topical issues and new trends in humanities and health. 
Pensamiento crítico en los grados de ciencias de la Salud. La importancia de educar la percepción como vía para que el estudiante adquiera criterio.

Francesc Borrell i Carrió

\title{
PENSAMIENTO CRÍTICO EN LOS GRADOS DE CIENCIAS DE LA SALUD. LA IMPORTANCIA DE EDUCAR LA PERCEPCIÓN COMO VÍA PARA QUE EL ESTUDIANTE ADQUIERA CRITERIO.
}

\section{Francesc Borrell i Carrió}

\begin{abstract}
Resumen: El Pensamiento Crítico (PC) es una corriente filosófica que propone un conjunto de métodos para ponderar argumentos. Sin embargo, en la vida cotidiana, y muy en particular en la práctica clínica, tenemos que retrotraernos al momento en que se elaboran o emergen estos argumentos, eso es, debemos reflexionar críticamente sobre cómo percibimos al paciente y la situación clínica en su conjunto. No hay PC sin percepción crítica, e incluso más, la aportación de valor que realiza un clínico depende sobre todo de su mirada semiológica, que no es otra cosa que educar la percepción. Para lograrlo, el clínico tiene tres procedimientos: inmersión, imitación y contraste. Será sobre todo el contraste subjetivo la técnica que puede ayudarle más a construir lo que llamamos "independencia de criterio", esta capacidad para modificar diagnósticos y definir la mejor conducta a seguir. La independencia de criterio deberá complementarla con la interdependencia con la comunidad científica.
\end{abstract}

Palabras clave: Sentimiento crítico/percepción/ práctica clínica/semiologia

\begin{abstract}
:
Critical Thinking (CT) is a philosophical field that proposes a set of methods to weigh arguments. However, in everyday life, and very particularly in clinical practice, we have to go back to the moment in which these arguments are elaborated or emerge, that is, we must go back to how we perceive the patient and the clinical situation as a whole. There is no CT without critical perception, and even more, the contribution of value that a clinician makes depends, above all, on his semiological sight, which is nothing else than an educated perception. To achieve this, the clinician has three procedures: immersion, imitation and contrast. It will be above all the subjective contrast technique that can help more to build what we call "independence of criteria", this ability to modify diagnoses and define the best behavior to follow. Criterion independence should be complemented with the interdependence criteria with our scientific community.
\end{abstract}

Keywords: Critical feeling/ perception/ clinical practice/ semiology.

Artículo recibido: 21 noviembre 2018; aceptado: 8 marzo 2018.

\section{¿ES IMPORTANTE -O DEBIERA IMPORTARNOS- EL PENSAMIENTO CRÍTICO (PC) EN LA PRÁCTICA COTIDIANA DE LAS PROFESIONES SANITARIAS?}

Mucho. Abarca todas las fases de la reflexión ante un paciente, no importa si hablamos de enfermería, medicina, fisioterapia, odontología o psicología. Una buena formación en pensamiento crítico (PC) influye en la imagen que nos 
Pensamiento crítico en los grados de ciencias de la Salud. La importancia de educar la percepción como vía para que el estudiante adquiera criterio.

Francesc Borrell i Carrió

formamos del paciente y qué consejos o información le brindaremos. Permitidme algunos ejemplos que nos servirán, además, para comentar aspectos sustanciales del PC.

\section{PRIMER EJEMPLO}

Una enfermera realiza una cura y la paciente le dice: "la otra enfermera usa otro tipo de pomada..."

¡¡Ah!, ¿si? Bueno, ya sabe usted, cada maestrillo tiene su librillo...

\section{COMENTARIO}

La observación de la paciente podemos tomarla como una crítica, una llamada de atención o simplemente una reacción de sorpresa. La respuesta de la enfermera parece de tipo justificativo, por lo que no sería exagerado deducir que se ha sentido impugnada. Desde un análisis meramente de técnicas de comunicación correspondería lo que llamamos "respuesta evaluativa", del tipo:

- ¡Ah!, ¿si? ¿recuerda cómo era o se llamaba esta pomada?...

Desde el punto de vista del PC esta enfermera debería tener un hábito de reflexión que le llevara a pensar de forma casi automática:

- “ ¿podría darse el caso de que esta otra enfermera estuviera haciendo alguna técnica más actualizada o efectiva de la que yo hago?".

Este primer ejemplo nos muestra cómo una buena formación en comunicación ayuda, pero no es lo mismo, a una reflexión crítica. Entrenar a los profesionales en PC es ayudarles a crear hábitos automáticos de reflexión. En este caso concreto el hábito de reflexión consiste en estar siempre abierto a aprender de los demás, lo que también llamaremos en este artículo cultivar la interdependencia de criterio.

\section{SEGUNDO EJEMPLO}

- Dr., quisiera que usted me recetara Prozac...

- Aja.... ¿y qué le hace pensar que puede irle bien? 
Pensamiento crítico en los grados de ciencias de la Salud. La importancia de educar la percepción como vía para que el estudiante adquiera criterio.

Francesc Borrell i Carrió

- Pues verá, desde hace 4 semanas he perdido el apetito y estoy desmoralizada....... etc.

\section{COMENTARIO}

Este ejemplo parece de Perogrullo, pero no lo es. Tenemos fuertes evidencias del impacto que tienen las expectativas del paciente en las decisiones que toma el médico (1). La conversación es diferente a que hubiera empezado de esta suerte:

- Doctor vengo porque desde hace 4 semanas he perdido el apetito y estoy desmoralizada...

Es diferente porque al priorizar la expectativa de que se le prescriba un antidepresivo concreto induce en el médico pensamientos del tipo:

- El paciente cree tener una depresión.... Quizás ha tenido experiencias previas, propias o de gente cercana, en el sentido de que esta medicación le ha funcionado.... Si yo le doy Prozac quedará contenta.... Por otro lado, si cubro sus expectativas jugará a mi favor el efecto placebo...

Estos pensamientos deberá contrarrestarlos para lograr una decisión mejor ponderada, y puede que en su diálogo interno le diga algo así:

- Debo enfocar esta entrevista como si ignorara la petición de un antidepresivo para así lograr un juicio claro y no sesgado acerca de lo que le ocurre...

Imaginemos que transcurren 20 minutos de provechoso diálogo y el profesional cree que la paciente puede estar iniciando una depresión. Su diálogo interno quizás fuera algo así:

- Este paciente puede estar iniciando una depresión, pero en estas fases iniciales aún es pronto para realizar el diagnóstico. Simplemente le diré que vuelva dentro de un par de semanas a ver cómo evoluciona.

O bien:

- Parece que en efecto pudiera estar iniciando una depresión, pero en estas fases iniciales aún es pronto para realizar el diagnóstico. Ahora bien, si le digo 
Pensamiento crítico en los grados de ciencias de la Salud. La importancia de educar la percepción como vía para que el estudiante adquiera criterio.

Francesc Borrell i Carrió

que venga dentro de 2 semanas puedo perderla como paciente, pues una expectativa tan bien formulada indica que en cualquier caso espera una prescripción terapéutica (aunque no sea el Prozac). Si esta acción consistiera en darle Prozac tendría a mi favor el efecto placebo, pero la paciente también puede entender que podemos enmascarar otro tipo de enfermedad que se expresa de manera similar, como puede ser un hipotiroidismo. Por consiguiente, tengo dos retos: llegar a un diagnóstico no interferido por una medicación recetada de manera prematura, pero al mismo tiempo hacer partícipe de esta decisión a la paciente, pues de lo contrario irá a otro médico mas resolutivo, que sin dudarlo le dará el Prozac.

Observe el lector que en este segundo diálogo el médico incorpora PC:

1. Establece un marco de diagnóstico no influido por la percepción de la paciente: "puede que tenga depresión como que no la tenga, pero debo llegar a esta conclusión por mí mismo".

2. Pondera el tipo de mensaje a dar a la paciente en función de un cierto modelo de satisfacción: "ante una expectativa tan explícita se espera de mi algún tipo de prescripción terapéutica". No se limita a considerar "dar o no dar el antidepresivo", sino fomentar una relación de confianza que le permita afianzar dicha relación.

\section{¿EN QUÉ FASES DE LA ENTREVISTA CLÍNICA INTERVIENE EL PENSAMIENTO CRÍTICO?}

Sería un error pensar que el PC interviene sólo en el momento de reflexionar sobre el diagnóstico o el consejo a dar. E incluso más grave confundir PC con conocer y seguir las guías clínicas basadas en evidencias. EI PC interviene en el mismo momento en que nos formamos una imagen del paciente. He aquí de manera más clara los momentos claves en que el PC juega un rol determinante:

1. Percepción del paciente.

2. Calidad, verosimilitud y suficiencia de los datos obtenidos.

3. Interpretación de dichos datos. 
Pensamiento crítico en los grados de ciencias de la Salud. La importancia de educar la percepción como vía para que el estudiante adquiera criterio.

Francesc Borrell i Carrió

Existe un sesgo en la literatura especializada en PC en el sentido de otorgarle un papel casi exclusivo en el análisis argumental (2,3). Lo cierto es que no existe PC sin percepción crítica (4). En ocasiones el análisis de argumentos resulta del todo inútil porque se basan en asunciones equivocadas. En la actividad clínica evidente: en el ejemplo anterior, discutir si un paciente necesita o no necesita un antidepresivo resulta del todo improcedente si antes no tenemos una perspectiva adecuada de dicho paciente. Esta perspectiva en ocasiones puede basarse en una observación de cómo se mueve, como habla, y qué tipo de expresiones faciales cruzan su rostro, sus antecedentes patológicos, cómo vive, qué le preocupa... (sin olvidar, por supuesto, una valoración biológica y somática).

Educar la percepción significa dos cosas: dotarnos de una metodología y rectificar sesgos. Examinemos la metodología.

Los pediatras han introducido lo que llaman el "triángulo de valoración pediátrico", que consiste de visu en apreciar la apariencia del niño, como respira y posibles problemas cardiocirculatorios. Este mismo triángulo lo podemos aplicar a todas las edades, además de preguntarnos: ¿qué tipo de persona tengo delante? ¿qué puedo deducir de su comunicación verbal y no verbal? Para que se produzca una educación perceptiva es imprescindible dotarnos de una metodología de este tipo, siempre alertas a si alguien es más perceptivo que nosotros y, en tal caso, aprender de él o ella.

Sería de gran utilidad que en los Grados de Ciencias de la Salud los estudiantes vieran clips de video con pacientes - de variadas extracciones étnicas y sociales- expresando las quejas más variopintas. De tal manera podríamos entrenarlos en apreciar cambios sutiles en la manera de expresar dichas quejas. Históricamente -en asignaturas como propedéutica y semiología-, hemos dado una gran importancia a la manera de preguntar, logrando anamnesis exhaustivas y minuciosas; pero en ocasiones una perspicaz observación nos habría alertado sobre la escasa calidad de los datos que obteníamos. Por ejemplo: el paciente está desconcentrado. Sabemos preguntar, pero tenemos que aprender a escuchar. Lo que implica que en varios momentos el clínico se tiene que interrogar: 
Pensamiento crítico en los grados de ciencias de la Salud. La importancia de educar la percepción como vía para que el estudiante adquiera criterio.

Francesc Borrell i Carrió

- “¿estoy obteniendo los datos que voy a requerir para una buena cualificación del problema del paciente?, ¿son de calidad?”.

A este tipo de preguntas le llamamos "nuestro supervisor interno" (5). Porque sólo hay buenos diagnóstico si partimos de buenos datos de anamnesis y exploratorios, y obtenerlos supone controlar la calidad de estos datos antes de darlos por fiables.

Uno de los aspectos más delicados de la práctica clínica es precisamente escoger los datos relevantes para llegar a un diagnóstico (o cualificación del problema). Nos movemos en un entorno lleno de "ruido de fondo", por ejemplo:

- ¡No se puede imaginar la noche que he pasado! Ya sabe que tengo la espalda fatal, y no encuentro la posición adecuada. Para dormir tengo que encajarme entre 4 almohadas, y si como me ha pasado esta noche se me junta la tos $y$ ahogo.... Lo cierto es que tenía que incorporarme porque me ahogaba, y tenía que sentarme en la cama un buen rato... no podía en absoluto tumbarme. Al final me he dormido en el sillón de la cocina, y claro, esta mañana, no le digo como tengo la espalda, sencillamente destrozada.

Un clínico con experiencia rápidamente separa "lo nuevo" de "lo viejo", lo que pueden ser síntomas importantes, (disnea nocturna), de lo que son dolamas derivadas de procesos crónicos (dolor espalda). Es lo que llamamos "escucha semiológica". Un tipo de escucha que, superpuesta a la escucha de tipo social, se produce sólo si sabemos anular los prejuicios sobre "lo más plausible" que le esté ocurriendo al paciente. Imaginemos en el ejemplo anterior que pensáramos.... "¡Uf!, otra vez este paciente quejándose de la artrosis"... Este prejuicio haría casi inviable la escucha ex novo necesaria para darnos cuenta que el paciente está en un proceso de disnea paroxística nocturna. Detectar una disnea paroxística puede ser la diferencia, para el paciente, entre vivir o morir.

La exploración física no escapa a esta metodología. La educación perceptiva tiene ahí varios retos:

a) Distinguir lo normal de lo anormal (aunque no forzosamente patológico), incorporando variantes poco frecuentes de normalidad. Ejemplo: un nódulo de 
Pensamiento crítico en los grados de ciencias de la Salud. La importancia de educar la percepción como vía para que el estudiante adquiera criterio.

Francesc Borrell i Carrió

tiroides de apenas un centímetro de diámetro versus las inserciones musculares presentes en esta zona del cuello, o acúmulos de grasa (cuello consular, por ejemplo).

b) Determinar que una variable que cambia lentamente ha llegado a un punto crítico. Ejemplo: enlentecimiento de una curva de crecimiento en pediatría, o la pérdida gradual de peso de un anciano.

c) Descartar hallazgos que no aportan valor a efectos del diagnóstico principal. Sería en este caso el equivalente al "ruido de fondo" que mencionábamos para la anamnesis.

d) Valorar signos físicos que siempre equivalen a la presencia de una enfermedad relevante. Por ejemplo, una esplenomegalia, sea cual sea el contexto en que la descubrimos.

El tercer paso que reseñábamos es la interpretación. En realidad, durante todo el proceso de anamnesis y exploración física, ya hemos realizado muchas interpretaciones. Digamos que aquellas fueron interpretaciones muy a ras de suelo, del tipo: “¿tiene una auscultación cardíaca normal?”, “¿realmente le duele si le aprieto aquí?". Ahora sin embargo hay que ganar perspectiva: "con los datos que tengo, ¿qué le puede ocurrir a este paciente?"

En este punto la mente humana opera con abducciones, es decir, compara el cuadro clínico presente con cuadros clínicos formalizados, "idealizados"(6,7,8). Más o menos la manera de razonar seria la siguiente:

- "Este paciente tiene dolores y debilidad de hombro de 3 meses de evolución, un cuadro que puede coincidir con polimialgia reumática, pero en su caso la velocidad de la sangre es de 40 , en el límite para un diagnóstico confiable... quizás tenga otro padecimiento, como un trastorno derivado de un ejercicio intenso, o quizás.... Etc.".

La experiencia clínica juega un papel relevante en los procesos de abducción. Un especialista en polimialgia reumática necesita apenas unos minutos para distinguir este cuadro de una artrosis o algún tipo de colagenosis. Si las pruebas de laboratorio no van a favor se llevará una sorpresa mayúscula, pues simplemente con los datos clínicos ya había alcanzado el punto de certeza. Y a diferencia de un 
Pensamiento crítico en los grados de ciencias de la Salud. La importancia de educar la percepción como vía para que el estudiante adquiera criterio. Francesc Borrell i Carrió

médico novato, dará tanta importancia a esta orientación diagnóstica basada exclusivamente en los datos de anamnesis y exploratorios como a los obtenidos mediante laboratorio o exploraciones radiológicas.

La segunda tarea que deberá afrontar el clínico es compensar sesgos de percepción. El tema es muy extenso, pero abarca aspectos como los siguientes:

- Estar de buen humor (o mal humor) tiene efectos sobre las decisiones que tomamos. $\mathrm{Y}$ no sólo las decisiones, también tiene efectos sobre la manera como percibimos el mundo.

- Una vez hemos tomado una decisión somos maestros en justificarla; preferimos equivocarnos a reconocer que nos hemos equivocado.

- Un carácter impulsivo nos lleva a tomar decisiones precipitadas. Nos ponemos nerviosos y preferimos arriesgarnos a tomar una decisión equivocada a solicitar más opiniones o ponderarlas de manera mas pormenorizada. Aplíquese la misma advertencia para otros rasgos de carácter, como un carácter irritable, o ansioso, u obsesivo...

- Muchas veces adornamos con argumentos decisiones que hemos tomados sencillamente porque nos complacían a nivel emocional. Esto es así sobre todo cuando nuestro grupo de referencia espera de nosotros que hagamos $u$ opinemos en un sentido determinado.

El siguiente ejemplo ilustra este sesgo gregario:

\section{TERCER EJEMPLO:}

- Doctor, vengo para que me renueve estas pastillas del Parkinson. Ya hace más de 5 años que las tomo.

- Aja.... Vamos a verle andar.....

\section{COMENTARIO}

El médico ha observado que los movimientos del paciente no se corresponden con una enfermedad de Parkinson de más de 7 años de evolución. Por este motivo decide explorarle y verifica que en efecto no hay temblor, ni falta de balanceo, y el tono muscular es prácticamente normal. El diagnóstico lo estableció 
Pensamiento crítico en los grados de ciencias de la Salud. La importancia de educar la percepción como vía para que el estudiante adquiera criterio.

Francesc Borrell i Carrió

un prestigioso neurólogo, lo que no es óbice para que proponga al paciente una progresiva disminución del tratamiento y una nueva remisión al especialista para reconsiderar el diagnóstico.

Pocas situaciones en la clínica diaria exigen más independencia de criterio que revertir un diagnóstico formulado por otros colegas, sobre todo si procede de un estamento que ostenta más autoridad. Sería el caso de un diagnóstico reconsiderado por una enfermera, o un diagnóstico reconsiderado por un médico de familia (en relación a un especialista). Desde luego no hay "premio" corporativo a tal hallazgo, todo lo contrario, y quizás el único agradecido sea el del propio paciente. Todo lo cual nos lleva a qué tipo de valores gregarios se enfrenta el PC, tema que desarrollaremos a continuación. Pero antes hagamos un breve resumen de donde se posiciona el PC en estas fases de la entrevista:

\section{PERCEPCIÓN DEL PACIENTE}

- ¿Qué tipo de persona tengo delante mío? PC: contrarrestar estereotipos.

- Lectura estructural y dinámica: triángulo de observación, comunicación verbal y no verbal. PC: educación perceptiva, segregar "lo nuevo" de lo viejo, percatarnos de cosas que nadie antes había detectado.

\section{INTEGRAR DATOS}

- Calidad de estos datos, verosimilitud.

- ¿Dónde debo focalizar la búsqueda?

\section{INTERPRETAR CON PERSPECTIVA BIOGRÁFICA}

Por consiguiente, el PC emerge en todas estas fases anulando los prejuicios: preguntándose si aprovechamos todos los datos "crudos", derivados de nuestra percepción directa, y si los datos que obtenemos mediante la anamnesis y la exploración física tienen suficiente calidad, si son verosímiles.

\section{INDEPENDENCIA DE CRITERIO Y VALORES GREGARIOS}

Como apuntábamos más arriba, el PC se alimenta de una percepción educada e independiente, una percepción pues que no teme enfrentarse a la opinión 
Pensamiento crítico en los grados de ciencias de la Salud. La importancia de educar la percepción como vía para que el estudiante adquiera criterio.

Francesc Borrell i Carrió

de otros profesionales. Pero para llegar a este punto, lógicamente el profesional tiene que empoderarse. Poco a poco tiene que ganar en confianza, compararse a los mejores y persuadirse de que fue capaz de escuchar un roce pleural, o un chasquido mitral. Eso lleva años de concienzuda disciplina. Pero nada de ello es posible si no logra lo que llamamos independencia de criterio.

Estamos acostumbrados a cierta cultura grupal que favorece la lealtad por encima de la verdad, sobre todo la lealtad al líder o a lo que el grupo decide que es "verdad", y a identificar lo que creemos con lo que somos. Cuando se rebaten unas creencias tal, parece que se impugne a la globalidad de la persona.

Por otro lado, aún persiste la imagen del clínico sabelotodo capaz de formular diagnósticos de enfermedades raras gracias a su "buen ojo clínico". Nada de eso existe. Los buenos diagnósticos son los que sirven a los pacientes. $Y$ pensar en diagnósticos improbables solo está justificado cuando no encajan los más comunes. Un aforismo clásico pone el dedo en la llaga: "antes que pensar en un diagnóstico raro, hay que pensar en presentaciones poco frecuentes de enfermedades comunes".

El clínico joven tiene que aprender a tener "su" opinión, a sustentarla sin dogmatismos, dispuesto a rectificarla si con ello gana una perspectiva más acorde a la realidad... Son hábitos totalmente contrarios a cierta cultura de la testarudez, a este "querer tener razón" para asentar cierta autoridad en el grupo.

¿Cómo deberían llegarnos los jóvenes bachilleres, tras su paso por unas aulas en las que se hubiera priorizado el PC? Un joven de este tipo nos diría frases tales como:

1. No me importa tener o no tener razón, lo que quiero es aproximarse a como son de verdad los problemas.

2. Hay dos tipos de conocimiento, las basadas en una creencia (=K1) muchas veces transmitida por una autoridad, y las basadas en un modelo de cómo funcionan las cosas $(=\mathrm{K} 2)$. Lo ideal sería tener pocas de las primeras y muchas de las segundas. Ejemplo: "una fiebre de más de $39^{\circ}$ es muy mala" (=K1) versus "la fiebre es una defensa contra la infección, y está bien tenerla salvo que el niño lo veas muy amodorrado" (= K2). 
Pensamiento crítico en los grados de ciencias de la Salud. La importancia de educar la percepción como vía para que el estudiante adquiera criterio.

Francesc Borrell i Carrió

3. No siempre tengo que dar mi opinión, sobre todo en temas que desconozco en profundidad. Pero incluso en temas que he estudiado procuro expresar mis opiniones de manera probabilística, (por ejemplo: "por lo general", "a menudo", "pocas veces"...).

4. Tenemos tendencia a ver los conflictos en blanco y negro (eso es, como dilemas), cuando por lo general hay una amplia gama de grises. Explorar esta gama de grises significa que podemos encontrar salidas más airosas a problemas aparentemente irresolubles.

5. No soy lo que creo. Resulta un trágico error basar mi auto-estima en querer tener razón, o en querer demostrar a los demás que mis creencias son las buenas. Sobre todo porque quienes me rodean me aprecian por otras razones (mi manera de ser, mis actos), no porque todas mis creencias sean (o me imponga el deber de que sean) verdades.

6. Solo se aprende PC con rectificación. Y rectificar siempre es un ejercicio de humildad. Experimentar dolor cuando rectificamos es absolutamente normal, y debo acostumbrarme a ello si quiero avanzar. Por consiguiente, analizo lo que siento en el acto de rectificar, analizo las resistencias que opongo a un cambio, sobre todo cuando este cambio es de actitud, o tiene implicaciones emocionales. Por ejemplo: un paciente que me cae mal y debo esforzarme en identificar el porqué de esta animadversión. Muchas veces puede deberse a un detalle de comunicación o de apariencia y, por consiguiente, debo reconocer lo muy equivocado/a que estoy. Es un acto de humildad, y como tal me cuesta realizarlo. Pero en la medida en que lo voy poniendo en práctica, cada vez me cuesta menos.

\section{ENSEÑAR PENSAMIENTO CRÍTICO EN LOS GRADOS DE CIENCIAS DE LA} SALUD

En los grados de Ciencias de la Salud se enseña Medicina Basada en la Evidencia (MBE). La MBE es una metodología que reflexiona sobre el grado de verosimilitud de afirmaciones de tipo diagnóstico, preventivo o terapéutico. La MBE forma parte del PC, pero tal como decíamos previamente es un componente más que no debe desviarnos del objetivo principal: educar la percepción del estudiante. 
Pensamiento crítico en los grados de ciencias de la Salud. La importancia de educar la percepción como vía para que el estudiante adquiera criterio. Francesc Borrell i Carrió

Por más evidencias que un estudiante conozca de poco le servirá si no desarrolla su propio criterio ante cada uno de sus pacientes. Si no sabe pensar por sí mismo.

Por consiguiente, estamos hablando de que un joven de unos 20 años más o menos, tendrá que educar su manera de ver a otras personas (los pacientes), a través de un filtro, la mirada semiológica. Esta manera de mirar tiene que llevarle a decisiones tan importantes como: “¿estoy frente a una persona enferma, o sólo con alteraciones funcionales que no requieren medidas especiales?, ¿está triste o tiene un síndrome depresivo?, ¿es un picor normal o es una enfermedad de la piel?, etc."

¿Cómo educamos la percepción de este estudiante? ¿Cómo fomentamos que cada estudiante edifique su "propio criterio", para responder a preguntas como las anteriores?

La percepción del estudiante de enfermería o medicina se educa de manera similar a cómo educa su percepción un estudiante de música: mediante inmersión, imitación y contraste.

\section{INMERSIÓN}

En esta fase el estudiante identifica ruidos cardiopulmonares, maneras como los pacientes expresan el dolor, morfología de diferentes tipos de lesiones, etc. Por fortuna hoy en día los estudiantes tienen simuladores de ruidos, amplias bibliotecas de imágenes y en ocasiones laboratorios de habilidades clínicas. Estos instrumentos le facilitan la lectura de signos semiológicos, y su principal reto será asociar estos signos físicos a entidades mórbidas.

\section{IMITACIÓN}

Las prácticas en servicios hospitalarios, centros de salud y urgencias le tienen que permitir al estudiante observar a un experto realizar y valorar maniobras exploratorias. Resulta del máximo interés que observe diferentes personas con diferentes estilos.

\section{CONTRASTE}

Esta fase resulta crucial; en ella es el estudiante quien realiza las maniobras exploratorias y tiene que sacar sus propias conclusiones. Idealmente un tutor tiene 
Pensamiento crítico en los grados de ciencias de la Salud. La importancia de educar la percepción como vía para que el estudiante adquiera criterio.

Francesc Borrell i Carrió

que supervisarle y proporcionarle un feedback inmediato. Es lo que llamamos "tutorización directa". Por desgracia el estudiante pocas veces es supervisado de esta manera.

Para enfatizar este punto imaginemos las siguientes escenas:

A. El tutor le pide al estudiante que realice una anamnesis a un paciente geriátrico. Anamnesis que debe incluir un Mini Mental State (MMS). Después de que el estudiante ha realizado la tarea repasan el resultado del MMS con el tutor.

Comentario: esta tarea obliga al estudiante a imitar y poner en práctica conceptos que quizás ha aprendido, pero no incluye tutorización directa. Si el tutor estuviera escuchando desde la otra habitación cómo interactúa con el paciente, y después le proporciona feedback, en tal caso sí incorporaría el contraste. Idealmente el tutor debería proporcionar retroacción en momentos clave de la entrevista, justo después de que se hubieran producido interacciones con alto valor pedagógico. Por ejemplo: "Perdone Sr. García que le robe a Sandra (estudiante enfermería) unos instantes (en la otra habitación) Sandra, ¿crees que el paciente ha entendido el por qué le preguntas acerca del día de la semana y dónde se encuentra? Deberías introducir el sentido de este test. ¿Cómo lo harías? Muy bien, pues vuelve con el paciente y a ver ahora cómo lo haces".

B. El tutor solicita al estudiante que realice una exploración de rodilla dolorosa, y está presente mientras el estudiante realiza las maniobras. Al finalizar esta exploración le solicita al estudiante a qué conclusiones ha llegado.

Comentario: aunque el formato escogido parece de tutorización directa, no lo es. El tutor debería rectificar al estudiante en cada detalle exploratorio, proporcionándole un feedback inmediato. Por ejemplo: "muy bien la maniobra de Clark, pero en el caso del McMurry observa cómo lo hago yo ahora y lo repites". "Este gesto hazlo con mucho cuidado, pues puedes hacer daño al paciente. Usted Sr García se queja si le 
Pensamiento crítico en los grados de ciencias de la Salud. La importancia de educar la percepción como vía para que el estudiante adquiera criterio.

Francesc Borrell i Carrió

hacemos daño, ¿de acuerdo?” (dirigiéndose al estudiante): “¿crees que esta maniobra es normal? ¿revela que pudiera tener algo de derrame sinovial?”.

Por supuesto el contraste no sólo se realiza en el marco de esta tutorización directa. La metodología de portafolio nos ofrece la oportunidad de practicar el contraste perceptivo con gran profundidad. He aquí mi experiencia al respecto tal como lo explico al estudiante:

"Ahora me interesa que escojas una de las entrevistas clínicas que vas a presenciar y tomes notas lo más precisas posible. Me interesa, en primer lugar, que expliques el contexto de la entrevista, edad y sexo del paciente, etc. Luego, las diferentes interacciones verbales, pero sobre todo con los componentes emocionales y de comunicación no verbal. Observa cuales son en cada momento las intenciones que guían al paciente y que me guían a mí. Mañana me traerás la entrevista y la comentaremos. ¿de acuerdo?"

Esta entrevista puede haberse video-grabado con permiso del paciente, lo que añade una instancia "objetiva" para verificar las observaciones del estudiante, pero también puede realizarse sin dicha grabación. El lector puede imaginar la riqueza de contenidos que pueden extraerse. Por ejemplo:

"tienes mucha razón al apuntar que esta intervención del paciente me puso algo nervioso, por ello intenté desviar la conversación. No es casual que introdujera en este punto el tema de la medicación, pero observa como al poco rato vuelvo al tema que más me preocupa, etc."

Otro tipo de ejercicios que desarrolla la agudeza perceptiva consiste en plantear retos al estudiante. Por ejemplo:

"(Dirigiéndose al estudiante): ahora entrará un paciente y me interesa que apuntes en este papel qué edad le supones, qué peso y talla aproximados, y luego cuantos detalles consideres interesantes en relación a cómo se mueve, cómo anda, como se expresa, etc. Si te atreves apunta también qué problemas de salud piensas que puede tener, no importa si te equivocas. Puedes decir: <creo que tiene un problema reumático>, o bien ser más preciso, 
Pensamiento crítico en los grados de ciencias de la Salud. La importancia de educar la percepción como vía para que el estudiante adquiera criterio.

Francesc Borrell i Carrió

$<$ creo que tiene un problema de artrosis que le dificulta la marcha>. Una vez se haya marchado el paciente lo comentaremos, ¿te parece bien?"

Este tipo de ejercicios le hace ver al estudiante que tiene una idea alejada de la realidad sobre aspectos tan básicos como la edad y el peso de una persona, por no decir el tipo de marcha, o la identificación de cadencias verbales. Desde luego un ejercicio de este tipo activa enormemente su atención. Finalmente debemos proporcionarle al estudiante instrumentos para que de manera autónoma desarrolle sus capacidades de percepción crítica. Esta metodología la llamo "doble contraste subjetivo" y consiste en lo siguiente... (Simularé una conversación introductoria a este método con un alumno):

- "Ya has acabado tus prácticas con nosotros y quisiera darte una pauta de trabajo para que desarrolles tus habilidades de exploración, tanto exploración física como de anamnesis. Se llama metodología del doble contraste. Verás, un clínico está permanentemente madurando sus capacidades. Cuando deja de hacerlo estas habilidades decaen, no importa lo bueno que hubiera sido previamente en esta materia. ¿Cómo podemos ampliar y avanzar en nuestra capacidad de explorar bien? Antes de realizar cada maniobra exploratoria pregúntate: ¿qué hallazgo encontraría en caso de que esta maniobra fuera anormal? Por ejemplo, en caso de derrame de rodilla encontraría que los dedos se me separan de la interlínea articular. Pues bien, mientras realizas la maniobra tienes que comparar lo que ocurre con este patrón que acabas de definir. $Y$ preguntarte si lo que experimentas es suficiente como para decir qué es anormal Este sería el primer contraste subjetivo.... ¿me sigues?"

- "Vamos ahora por el segundo contraste subjetivo. Imagina que has visitado a un paciente con un cuadro de infección respiratoria aguda. La exploración ha sido anodina, pero en la radiografía de tórax hay un infiltrado. He aquí una excelente oportunidad de aprender. Tienes que repetir la exploración pulmonar, (auscultación, percusión, transmisión de la voz, etc.) y ver si puedes apreciar algo que no hubieras valorado previamente. Otra situación: un colega ha descubierto un nódulo de tiroides que a ti se te había pasado 
Pensamiento crítico en los grados de ciencias de la Salud. La importancia de educar la percepción como vía para que el estudiante adquiera criterio.

Francesc Borrell i Carrió

por alto. ¿Qué sabe hacer este colega que tú no sabes hacer? Tienes que averiguarlo y si puedes, imitarlo, hasta lograr ser tan bueno como él o ella. En definitiva, este segundo contraste consiste en aprovechar lo que algo o alguien te están mostrando de un paciente y tú no habías sido capaz de detectar. El propósito es objetivizar qué habilidades puedes mejorar".

\section{ESPECIAL CUIDADO CON EL PROFESOR DE PRÁCTICAS}

Un aspecto por lo general bastante abandonado en los grados de Ciencias de la Salud es la relación entre los profesores que imparten las asignaturas y los profesionales (a los que llamaremos "profesores de prácticas") que acogen a los estudiantes en los servicios hospitalarios y centros de salud. Los estudiantes van a observar con mucho detalle cómo actúan "en el mundo real" estos profesionales, y contrastarán los mensajes recibidos en las aulas con la conducta de estos profesores de prácticas.

Un ejemplo demostrativo de lo anterior es el asombro que les causa a los estudiantes comprobar que los médicos no realizan habitualmente una exploración neurológica sistemática, con pares craneales incluidos, cuando un paciente acude a la consulta por cefalea. Otras veces se sorprenden porque el profesional se ha saltado algún paso en la anamnesis o la exploración física, y perciben que ellos lo hubieran hecho mejor. En ocasiones los estudiantes acaban de pasar por un servicio hospitalario y su perplejidad se debe a que en los centros de salud se solicitan menos exploraciones complementarias ante síntomas parecidos.

Para legitimar la práctica clínica real, el estudiante debe adquirir un enfoque bayesano. Es bien sabido gracias al Teorema de Bayes que la prevalencia pre-test condiciona el rendimiento de cualquier prueba diagnóstica. Por consiguiente, el clínico debe pasar por el cedazo de una buena anamnesis y exploración física a sus pacientes y solicitar pruebas a aquellos que superen este filtro, de lo contrario se expone a cosechar muchos falsos positivos. Pero eso no es todo. El enfoque bayesano nos lleva a aplicar nomogramas y tablas de riesgo que, al final, indican para cada persona el riesgo concreto que tiene de estar en una determinada situación clínica (por ejemplo, tener un cáncer de próstata). También este enfoque nos permite introducir el concepto de valor predictivo negativo. Todo ello resulta de 
Pensamiento crítico en los grados de ciencias de la Salud. La importancia de educar la percepción como vía para que el estudiante adquiera criterio.

Francesc Borrell i Carrió

gran interés para que el estudiante perciba la base racional de las decisiones que se producen en contextos clínicos diferentes al hospitalario.

No acaba aquí la tarea del profesor. Un aspecto olvidado es mantener una comunicación estrecha e intensa con los profesionales que acogen a los estudiantes. Estos profesionales deben ser escogidos por su buen hacer y porque entiendan la importancia de formar a estos jóvenes. Pero además tienen que recibir una formación específica para que sean capaces de realizar este contraste perceptivo que tan importante va a resultarle al estudiante. En este sentido realizamos en el Grado de Medicina con mi equipo $\left(^{*}\right)$ unas sesiones dirigidas a los profesores que tienen los siguientes contenidos:

- Qué espera el estudiante de su rotación. Objetivos de dicha rotación.

- Situaciones en las que el estudiante puede abandonar su rol de observador y realizar tareas. Como supervisamos dichas tareas. Concepto de tutorización directa. Ejemplos.

- Situaciones conflictivas y cómo resolverlas. A tal efecto solicitamos a los estudiantes que al finalizar su rotación cumplimenten un portafolio. En este portafolio se les pide, entre otras tareas, que describan de la manera más exacta posible una entrevista clínica. Este ejercicio nos da una idea de las situaciones problemáticas, así como los conflictos que presencian los estudiantes, y las técnicas de resolución empleadas por sus profesores de prácticas.

Cada año seleccionamos -a partir de estos portafolios- situaciones particularmente interesantes, descritas por los estudiantes, y las devolvemos a los profesores de prácticas en forma de sesiones clínicas. Estas sesiones resultan críticas para dichos profesionales, en primer lugar, porque se percatan de aspectos que quizás ellos valoran poco (y sí valoran los estudiantes); pero sobre todo porque tienen la oportunidad de analizar hasta qué punto la presencia del estudiante influye en la toma de decisiones, y a su vez poder ensayar nuevos enfoques para solventar situaciones conflictivas. 
Pensamiento crítico en los grados de ciencias de la Salud. La importancia de educar la percepción como vía para que el estudiante adquiera criterio.

Francesc Borrell i Carrió

\section{EL TRABAJO EN GRUPO}

El trabajo en grupo (de los estudiantes) es otra oportunidad para desarrollar la educación perceptiva. Cuando hablamos de trabajo en grupo estamos pensando en grupos pequeños, de 3 ó 4 componentes. He aquí la metodología básica desarrollada con mi equipo(*):

1. Aleatorizar la composición de los grupos. Es importante que todos sus miembros estén en pie de igualdad, de manera que, si 3 de sus miembros son amigos, van a dejar de lado al cuarto miembro. Además, resulta interesante que se conozcan poco, ya que las relaciones previas pueden condicionar el debate.

2. Es importante que los grupos tengan muy claro qué se les pide. Si por ejemplo les pedimos que interpreten un hemograma, el profesor resolverá un ejercicio similar para que los participantes sepan qué tipo de respuesta se espera de ellos.

3. Cada reto deben resolverlo en una primera instancia de manera individual, y sólo tras unos minutos (suficientes para la tarea), les permitiremos que lo compartan con el grupo. De esta manera preservamos su auto-estima (nadie está obligado a comunicar al grupo su respuesta), pero a su vez pueden compararse con el resto de miembros.

4. Los grupos perfeccionan entre todos sus miembros la respuesta al reto que les hemos planteado. A continuación, se les proporciona la respuesta y son ellos mismos quienes pueden puntuarse.

5. Las tareas que les proponemos son muy variadas: desde interpretación de datos de laboratorio, imágenes de exploraciones radiológicas 0 de dermatología, clips de vídeo en los que se plantean diferentes situaciones y retos, o escenificaciones que ellos mismos representan. En este caso proporcionamos una guía muy clara de lo que se pretende con cada situación. La tarea que debe desarrollar el estudiante que realizará el rol de profesional, y cómo deberá actuar el estudiante que representará al paciente. Muchas veces separamos a los estudiantes que harán el rol de pacientes y en un aparte fuera del aula les explicamos de manera pormenorizada algunos detalles de cómo deben actuar. Uno de los miembros del grupo tiene una plantilla (tipo "check 
Pensamiento crítico en los grados de ciencias de la Salud. La importancia de educar la percepción como vía para que el estudiante adquiera criterio.

Francesc Borrell i Carrió

list") con los parámetros fundamentales a observar. Este tipo de plantilla le habilita para dar una retroacción detallada a sus compañeros.

\section{INDEPENDENCIA E INTERDEPENDENCIA DE CRITERIO.}

En este punto resulta interesante enfatizar que a lo largo de nuestro trabajo apenas hemos hablado de MBE, sino de educar la percepción. ¡Qué tremendo error pensar que un experto en MBE es alguien que automáticamente aplicará el PC a su práctica clínica! Imaginemos un estudiante que sólo supiera MBE, incluso que tuviera un grado de conocimiento de las guías clínicas extraordinario ipero nada más que eso! ¿Podría ejercer como médico? La respuesta es que no.

Imaginemos un paciente diabético que inicia un dolor precordial al esfuerzo. Este médico observaría el Historial Clínico y en el mejor de los casos sabría que está frente a un paciente diabético con una estimación de riesgo cardiovascular en absoluto alarmante. Según su Guía clínica no correspondería realizar ninguna actuación extra. Por supuesto el paciente le está describiendo síntomas anginosos, pero toda vez que en el historial otro médico ha escrito: "descarto angor y síntomas de insuficiencia cardíaca", y nuestro médico descarta tal posibilidad. Necesitaría que otro colega le dijera: "en efecto, estás frente a un paciente diabético tipo II que parece iniciar una clínica anginosa, aunque ninguna exploración complementaria o de anamnesis previa nos alertaba". Este médico, sin mirada semiológica, aplicaría con todo rigor la guía clínica de diabetes; perdiendo así una oportunidad de oro para avanzarse a una complicación cardiovascular.

La mirada semiológica es la que nos permite tener un criterio clínico "novedoso", disruptivo, incluso. ¿Qué es tener criterio? Es aseverar algo sobre un paciente (o un contexto clínico, si se prefiere), que supone (o implica) una conducta diagnóstica o terapéutica. El profesional clínico está radicalmente sólo en este acto intelectual. Va a ser él o ella la que digan... "esta lumbalgia entra dentro de la normalidad", o bien, "esta lumbalgia no es normal, y por consiguiente veo necesario realizar más exploraciones". Después serán otros profesionales los que desmentirán o confirmarán este criterio, pero en aquel instante asistencial toda la responsabilidad recae sobre sus hombros. 
Pensamiento crítico en los grados de ciencias de la Salud. La importancia de educar la percepción como vía para que el estudiante adquiera criterio.

Francesc Borrell i Carrió

Un profesional que no ha educado suficientemente su percepción puede encontrarse muy incómodo en determinadas áreas de la patología humana. Por ejemplo, un ginecólogo puede tener muchas dudas interpretando -en el caso de una lumbalgia- las maniobras radiculares (tipo lasegue y sus variantes). En cambio, tendrá pocas dudas orientando un dolor pélvico crónico. Cuando la mirada semiológica está bien asentada, los criterios emergen con facilidad, y acuden en auxilio del clínico de una manera natural, casi mágica. Pero no hay magia, sólo hay un esfuerzo constante por aproximarnos a la realidad tratando de que nuestra mirada semiológica no se altere, ya sea porque aquel día estamos cansados, pesimistas, o todo lo contrario, exaltados e impetuosos. La mirada semiológica es un epitelio delicadísimo que se redefine en cada acto clínico, y no lo olvidemos, que afecta por igual a nuestra capacidad de observar, escuchar, palpar u oler.

Esta mirada semiológica implica empoderamiento.... El estudiante tiene que hacer caso de sus propias impresiones si quiere empezar a educarlas. Imposible avanzar sin un tutor que le advierta de lo importante, de lo sutil, de lo accesorio. Pero una vez inicia su carrera profesional deberá practicar su independencia de criterio con una radical modestia. Deberá complementar su independencia de criterio con la interdependencia de criterio, muy alerta a lo que otros son capaces de ver (o hacer) y él o ella no. Es en este punto en que se incardina la MBE, como consenso de una comunidad alrededor de situaciones clínicas bien estandarizadas.

Hemos desarrollado en otra parte los conflictos de comunicación y éticos que las nuevas evidencias pueden suponerle a un profesional clínico riguroso y honesto consigo mismo (9). Por ejemplo: ¿qué ocurre cuando la guía clínica me indica que en este caso de faringo-amigdalitis no es necesario dar un antibiótico, pero tengo la convicción de que sí debería darlo? No insistiremos en ello, pero sí parece interesante señalar una idea final: la aportación de valor que un profesional clínico realiza sobre la vida de sus pacientes se nutre de ambas fuentes, lo que es capaz de "ver" aquí y ahora, y -sobre este escenario-, lo que la comunidad científica prescribe como mejor conducta. La maduración clínica en ningún caso es solipsista, sino que consiste en este movimiento que va de la independencia a la interdependencia de criterio, de la percepción semiológica a la estandarización de situaciones clínicas, de mi experiencia clínica a la experiencia clínica de toda la comunidad científica. 
Pensamiento crítico en los grados de ciencias de la Salud. La importancia de educar la percepción como vía para que el estudiante adquiera criterio.

Francesc Borrell i Carrió

NOTA. $\left(^{*}\right)$ Equipo docente de la Facultad de Medicina, Campus Bellvitge. Lo componen, aparte del autor de este artículo: Eva Peguero, Juan Jose Mascort, Vanessa Monforte, César Asenjo, Josep Lluis Ballvé.

\section{Francesc Borrell i Carrió}

Profesor Titular del Departament de Ciències Clíniques. UB.

Médico de Familiar. Grupo Comunicación y Salud de España.

\section{BIBLIOGRAFÍA}

1. Kravitz RL, Franks $P$, Feldman M, Meredith LS, Hinton L, Franz C, Duberstein P, Epstein RM. What drives referral from primary care physicians to mental health specialists? A randomized trial using actors portraying depressive symptoms. J Gen Intern Med. 2006;21(6):584-9.

2. Dwyer CP. Critical Thinking. Cambridge University Press. Cambridge 2017

3. Keith J. Holyoak, Robert G. Morrison (Ed) The Oxford Handbook of Thinking and Reasoning (Oxford Library of Psychology) Oxford U Press 2013

4. Reber R. Critical Feeling. Cambridge University Press. Cambridge 2016

5. Borrell F, Hernández-clemente JC, Reflexión en la consulta.- Rev Clin Esp. 2014;214(2):94-100

6. Charlin B, Boshuizen HP, Custers EJ, Feltovich PJ. Scripts and clinical reasoning. Med Educ. 2007 Dec;41(12):1178-84.

7. Schmidt HG, Rikers RM How expertise develops in medicine: knowledge encapsulation and illness script formation. Med Educ 2007 Dec;41(12):1133-9.

8. Borrell, F. \& Hernández-Clemente, J., "La certeza diagnóstica. El difícil camino que nos lleva a comprender el concepto de probabilidad en la práctica clínica", en Revista Folia Humaníst 2016; 3: 27-41 --

Accesible: http://revista.fundacionletamendi.com/index.php/ficha/93/La-certeza-diagnstica

9. Borrell-Carrió F, Estany A, Platt FW, Morales-Hidalgo V. Doctors as a knowledge and intelligence building group: pragmatic principles underlying decision-making processes. $J$ Epidemiol Community Health 2015;69:4: 303-305

\section{Cómo citar este artículo:}

Borrell, F., "Pensamiento crítico en los grados de ciencias de la salud. Importancia de educar la percepción como vía para que el estudiante adquiera criterio", Folia Humanística, 2019 (11): 44-64. Doi: http://dox.doi.org/10.30860/0049.

(C) 2019 Todos los derechos reservados a la Revista Folia Humanística de la Fundación Letamendi Forns. This is an open access article. 